\title{
LINC00957 Acted as Prognostic Marker Was Associated With Fluorouracil Resistance in Human Colorectal Cancer
}

\author{
Li Hua Zhang ${ }^{1,2,3+}$, Long Hai Li ${ }^{1,3+}$, Peng Fei Zhang ${ }^{1,2 \dagger}$, Yan Fei Cai ${ }^{2}$ and Dong Hua ${ }^{1,2,3 *}$ \\ ${ }^{1}$ Affiliated Hospital of Jiangnan University, Jiangnan University, Wuxi, China, ${ }^{2}$ School of Pharmaceutical Science, Jiangnan \\ University, Wuxi, China, ${ }^{3}$ Wuxi Medical College, Jiangnan University, Wuxi, China
}

Colorectal cancer (CRC) is one of the most prevalent digestive tumors in China. Recent studies indicate that long intergenic non-coding RNAs (lincRNAs) play a crucial role in predicting survival for CRC patients. However, the novel lincRNA, LINC00957, is largely unclear in CRC. The purpose of the current study was to determine LINC00957 expression, assess its the clinical significance and explore the potential mechanism in CRC. The qRT-PCR was used to quantify the expression levels of LINC00957 in tissues and cell lines. Our research revealed that LINC00957 was significantly higher expression in CRC. In addition, the LINC00957 expression was associated with TNM stage and chemotherapy outcome, but age, gender, tumor size, histological grade, primary tumor location. CRC patients with high LINC00957 expression level showed poor overall survival $(P=0.002)$. Multivariate survival analysis indicated that LINC00957 was a prognostic factor for CRC patients $(P=0.010)$. Mechanically, inhibition of LINC00957 expression reversed 5-FU resistance by down-regulating P-gP. In summary, our study indicated that this novel IncRNA expression signature might be a useful biomarker of the prognosis and therapeutic target for CRC patients.

Keywords: LINC00957, colorectal cancer, drug-resistance, survival, lincRNA, TCGA, GSEA

tThese authors have contributed equally to this work

Specialty section: This article was submitted to Cancer Genetics, a section of the journal

Frontiers in Oncology

Received: 11 April 2019

Accepted: 31 July 2019

Published: 21 August 2019

Citation:

Zhang LH, Li LH, Zhang PF, Cai YF and Hua D (2019) LINC00957 Acted as Prognostic Marker Was Associated With Fluorouracil Resistance in Human

Colorectal Cancer

Front. Oncol. 9:776.

doi: 10.3389/fonc.2019.00776

\section{INTRODUCTION}

Colorectal cancer (CRC) has been reported to be one of the most common malignant tumors in the digestive system, with a high recurrence and cancer-related mortality rate worldwide (1). In China there were about 376,000 newly diagnosed colorectal cancer patients in 2015, leading to about 191,000 deaths (2). Despite wide application of standardized surgical treatment and better perioperative care, the treatment of CRC at early stages remains a challenge $(3,4)$. Therefore, there is an urgent need to seek for new biomarkers for early diagnosis and prognoses in patients with CRC and to develop potential targets for the therapeutic treatment. To better understand the mechanism of CRC, non-coding RNAs have been recently studied in CRC such as IncRNAs and lincRNAs. Long non-coding RNAs (lncRNAs) are aberrantly expressed in a broad spectrum of cancers, and they play key roles in promoting and maintaining tumor initiation and progression, demonstrating their clinical potential as biomarkers and therapeutic targets (5-8). Long intergenic non-coding RNAs (lincRNAs) are defined as autonomously transcribed non-coding RNAs longer than 200 nucleotides that do not overlap annotated coding genes, as future cancer biomarkers $(9,10)$. More 
and more lincRNAs have been found that they played important roles in CRC such as LINC00261, $\operatorname{LINC01420}(11,12)$. Recent studies indicate that long intergenic non-coding RNAs (lincRNAs) play a crucial role in predicting survival for CRC patients such as linc-UBC1 (13) and linc-UFC1 (14). However, the novel molecule, LINC00957, in CRC is still unclear.

In this study, we explore its function in CRC. Firstly, we analyzed lincRNA expression profiles in 440 CRC patients from The Cancer Genome Atlas (TCGA) and identified it was significantly associated with survival. Then we further examined the expression of LINC00957 in CRC tissues and cell lines compared to their matched adjacent non-tumor by qRT-PCR. Besides, we also analyzed the association between the LINC00957 expression and the clinic pathological characteristics of CRC patients. Mechanically, we explored that its function in 5-FU resistance was associated with multi-drug resistance relative genes. All data demonstrated that it was up-regulated in CRC, and was associated with TNM stage, chemotherapy outcome, but not with the age, gender, tumor size, histological grade, and primary tumor location. What's more, the high expression of LINC00957 suggested poor survival in CRC and involved in drug-resistance by regulating P-gp expression. Taken together, our study suggested that the upregulation of LINC00957 played a promoting role in CRC and it would be a potential biomarker for CRC progress and therapeutic target of CRC.

\section{MATERIALS AND METHODS}

\section{Patient Samples}

one hundred and sixty pairs of CRC tissues and adjacent normal tissues involved in present study were collected from Affiliated Hospital of Jiangnan University. Informed consents to approve our use of these tissues in the research were obtained from all patients. Patients who postoperatively received 5-Fu-based first-line systematic chemotherapy were enrolled in the present study. The protocol was approved by Affiliated Hospital of Jiangnan University. All methods involving human patients were performed in accordance with the relevant guidelines and regulations of Affiliated Hospital of Jiangnan University. These specimens were confirmed by histopathological examination and immediately stored at $-80^{\circ} \mathrm{C}$ until use.

Tumor assessment was performed after every 2 cycles of chemotherapy according to the Response Evaluation Criteria in Solid Tumors 1.1 (RECIST 1.1) criteria (22), and the assessment was classified as a complete response (CR), a partial response $(\mathrm{PR})$, stable disease $(\mathrm{SD})$ and progressive disease $(\mathrm{PD})$.

\section{Cell Lines and Cell Culture}

Human CRC cell lines (HCT8, SW480, SW620, LOVO, and HCT116) and normal human colon epithelial cells NCM460 were purchased from Nanjing Musai Bio-Tech Co, Ltd (Nanjing, China). Fluorouracil (5-Fu)-resistant HCT-8 cells (HCT-8/5-Fu) (KG333) was purchased from Keygen Biotech Co. Ltd. 5-Furesistant LoVo cells ( $\mathrm{LoVo} / 5-\mathrm{Fu}$ ) were derived by treating LoVo cells with stepwise increasing concentrations of 5-Fu (Jinyao Amino Acid Co. Ltd, Tianjin, China) over 6 months. 5-Furesistant CRC cells were cultured as we reported previously
(15). All cells were cultured in DMEM (Life Technologies) supplemented with $10 \%$ fetal bovine serum (FBS; Invitrogen), $10 \%(100 \mu \mathrm{g} / \mathrm{mL})$ penicillin and $100 \mathrm{U} / \mathrm{mL}$ streptomycin at $37^{\circ} \mathrm{C}$ with $5 \% \mathrm{CO}_{2}$ in a humidified atmosphere.

\section{RNA Extraction and qRT-PCR Assay}

Total RNA was isolated from CRC and matched adjacent tissues by using Trizol Reagent (Life Technologies), according to the manufacture's protocol. The concentration and purity of total RNA were measured on a nanodrop spectrophotometer (Thermo Scientific, USA). For qRT-PCR, RNA was reverse transcribed to cDNA by using a PrimeScript RT reagent Kit (Takara, Dalian, China). qRT-PCR was performed using a SYBR_Premix ExTaqII kit (Takara, Dalian, China) in the CFX96 Real-Time PCR Detection System (Bio-Rad, Hercules, CA, USA) to determine the relative expression levels of target genes. The reaction conditions was $95^{\circ} \mathrm{C}$ for $5 \mathrm{~min}$, followed by 40 cycles of $95^{\circ} \mathrm{C}$ for $15 \mathrm{~s}$ and $60^{\circ} \mathrm{C}$ for $30 \mathrm{~s}$. The $2^{-\Delta \mathrm{Ct}}$ method was used to calculate expression levels and $2^{-\Delta \Delta C t}$ method was used to calculate relative expression level. GAPDH was used as an internal control (16). The sequences of qRT-PCR primers as follow Table 1.

\section{Cell Transfection}

LOVO/5-FU and HCT8/5-Fu cells on 50-70\% confluence were treated with LINC00957-siRNA1 (GGUGGAAGAGCUUGG GCGACA, UCGCCCAAGCUCUUCCACCUG), LINC00957siRNA2 (UUAAACCUCCCAUCAUCUGUG, CAGAUGAUG GGAGGUUUAACU), LINC00957-siRNA3 (AUCAUAACAU GGUGAAAGGCA, CCUUUCACCAUGUUAUGAUGC) and nontargeting siRNA (si-NC), respectively. Cells were transfected with siRNA by using Lipofectamine 4000 (Invitrogen, Carlsbad, CA, USA) according to the manufacturer's instructions. The cells were harvested for further assays $48 \mathrm{~h}$ after transfection. Expression of LINC00957 were determined by Real-time PCR. Three independent experiments were performed, and the data are expressed as the mean \pm standard error of the mean (SEM).

\section{CTB Assay}

These cytotoxic effects of 5-FU were evaluated at $48 \mathrm{~h}$ with the CTB (celltiter-blue, cell viability assay) assay. Cells $(1 \times$ $10^{5}$ cells $/ 200 \mathrm{ul}$ ) were seeded in 96-well plates and placed in an incubator at $37^{\circ} \mathrm{C}$ for $12 \mathrm{~h}$. The medium was then replaced with medium containing different concentrations of 5-FU. After $48 \mathrm{~h}$, the medium of each well was added with $10 \mathrm{uL}$ CTB. After incubation for $4 \mathrm{~h}$, the plates were read on a microplate reader. Fluorescence density at $560 \mathrm{~nm}$ excitation

TABLE 1 | Sequences of gene-specific primers used for RT-PCR.

Gene Forward Primer $\left(5^{\prime}-3^{\prime}\right) \quad$ Reverse Primer $\left(5^{\prime}-3^{\prime}\right)$

LINC00957 TCAAGGGCGGAGCAAACATC AGTITGCAAAGCCTTCCTGTG

ABCA1 CAGGCTACTACCTGACCTTGGT CTGCTCTGAGAAACACTGTCCTC

ABCG2 GTTCTCAGCAGCTCTTCGGCTT TCCTCCAGACACACCACGGATA

ABCC1 CCGTGTACTCCAACGCTGACAT ATGCTGTGCGTGACCAAGATCC

ABCC2 GCCAACTTGTGGCTGTGATAGG ATCCAGGACTGCTGTGGGACAT

GAPDH GCACCCTGGTCTGAGGTTAAT AGGAGTGGGAGCACAGGTAAG 
and $590 \mathrm{~nm}$ emission was determined for statistical analysis. Three independent experiments were performed, and the data are expressed as the mean \pm standard error of the mean (SEM).

\section{Western Blot Assay}

Cells grown in 6-well plates were washed with cold PBS for three times and scraped off the culture dishes and treated with 200 ul RIPA buffer containing protease inhibitors and were then transferred to $5 \mathrm{ml}$ tubes. Those tubes were remained in ice for $30 \mathrm{~min}$ to cells to be completely lysed. After a centrifugation at $13,500 \mathrm{rpm}$ for $15 \mathrm{~min}$ under $4^{\circ} \mathrm{C}$, the supernatants were collected and were subsequently denatured with $1 \mathrm{X}$ SDS protein loading buffer at $100^{\circ} \mathrm{C}$ in $10 \mathrm{~min}$. Proteins were loaded on $12 \%$ SDS-PAGE gels and transferred onto PVDF membranes. The membranes blocked with 5\% BSA in TBST at room temperature for $2 \mathrm{~h}$ and incubated with primary antibodies under $4{ }^{\circ} \mathrm{C}$ overnight. The primary antibodies anti- ABCA1 antibody (1:1000, ab18180, abcam) and anti-GAPDH (1:5000, ab8245, abcam)were used to detect the expression of the proteins. The antigen-antibody complexes were visualized by an enhanced chemiluminescent reaction. Protein bands were analyzed using Image J software (National Institutes of Health, Bethesda, MD). Three independent experiments were performed, and the data are expressed as the mean \pm standard error of the mean (SEM).

\section{Data Mining and Analysis}

The LINC00957 gene expression in different tissues was from The Genotype-Tissue Expression (GTEx) project (https://gtexportal.org/).

Data in OncoLnc from the website (www.oncolnc.org, date to September, 2018) was been used. Then all data for individual cancers from TCGA were downloaded.

\section{Gene Set Enrichment Analysis (GSEA)}

The RNA-sequencing V2 datasets and clinical data of STAD samples were downloaded using the Bioconductor/TCGA Biolinks function package from TCGA database (http://tcgadata.nci.nih.gov./tcga/). The STAD-associated gene clusters and pathways were identified in the c2.cp.kegg.v5.1.symbols.gmt data set from the Msig database using GSEA version 3.0. Enrichment analysis was performed with random combination number of 1,000 and false discovery rate (FDR) $<0.05$ as the criteria for significantly enriched genes. The gene sets were classified into the LINC00957 high- and low-expression groups based on its median expression level, and the effect of LINC00957 expression was analyzed.

\section{Statistical Analysis}

The expression levels of LINC00957 in CRC tissues were analyzed by unpaired $t$ test. The chi-square and $t$ tests were performed to assess the relationship between LINC00957 expression and clinicopathological features. The Kaplan-Meier method and log-rank test were used to evaluate and compare the prognosis of CRC patient. Univariate and multivariate cox proportional hazards analyses were used to analyze the independent prognostic factors for survival in CRC patients. RStudio and GraphPad Prism software package were used to perform statistical analysis. Overall survival (OS) was calculated using the Kaplan-Meier method, and the results of the analysis were considered significant in a log-rank test if $P<0.05$.

\section{RESULTS}

\section{LINC00957 Was Abnormal Expression in Different Tissues and Only Associated With Colon Cancer in Gastrointestinal Cancer}

In order to explore LINC00957 expression, GTEx database was used to present the gene expression in different human tissues. It was showed that LINC00957 was abnormal expression in different tissues as Figure 1A. In Gastrointestinal cancer, esophageal carcinoma and stomach adenocarcinoma was not associated with overall survival $(P>0.05$, Figures 1B,C). However, colon adenocarcinoma was associated with overall survival $(P<0.05$, Figure 1D).

\section{LINC00957 Was Upregulated in CRC Tissues and Cell Lines}

In this study, we firstly compared the expression of LINC00957 by qRT-PCR in 160 cases of CRC tissues and their matched adjacent non-tumor tissues. As shown in Figure 2A, we found that the expression of LINC00957 in CRC tissues were significantly higher than that in normal tissues $(P<$ 0.001). We further examined its expression in the normal human colon epithelial cell line NCM460 and CRC cell lines (HCT8,SW480,SW620,LOVO and HCT116). As shown in Figure 2B, those data indicated that the expression of LINC00957 were also increased in CRC cell lines when compared with that in NCM460 cells. What's more, the expression of LINC00957 in 5-FU resistant cell lines were significantly upregulated as showed in Figure 2C. Those results showed that the expression of LINC00957 was aberrant in tissues and cell lines. It indicated that the expression was associated with CRC and drug resistance.

\section{LINC00957 Expression Was Associated With TNM Stage and Chemotherapy Outcome of CRC}

To further reveal the role of LINC00957 in CRC, we analyzed the correlation between LINC00957 expression level and clinicopathological features. Patients with CRC were divided into high and low expression group by the mean expression level of LINC00957. Those data showed that the high expression group was significantly correlated with TNM stage $(P=0.040)$ and chemotherapy outcome $(P=0.026)$. However, the level of LINC00957 expression in CRC tissues was not associated with the age, gender, tumor size, histological grade, primary tumor location (all $P>0.05$, Table 2). These findings suggested that the increased expression of LINC00957 was involved in the malignant progression and chemotherapy resistance of CRC patients.

The relationship between the LINC00957 expression and the survival time of CRC patients was further analyzed. Our data showed that CRC patients with high LINC00957 expression appeared a worse prognosis when compared with those with low 


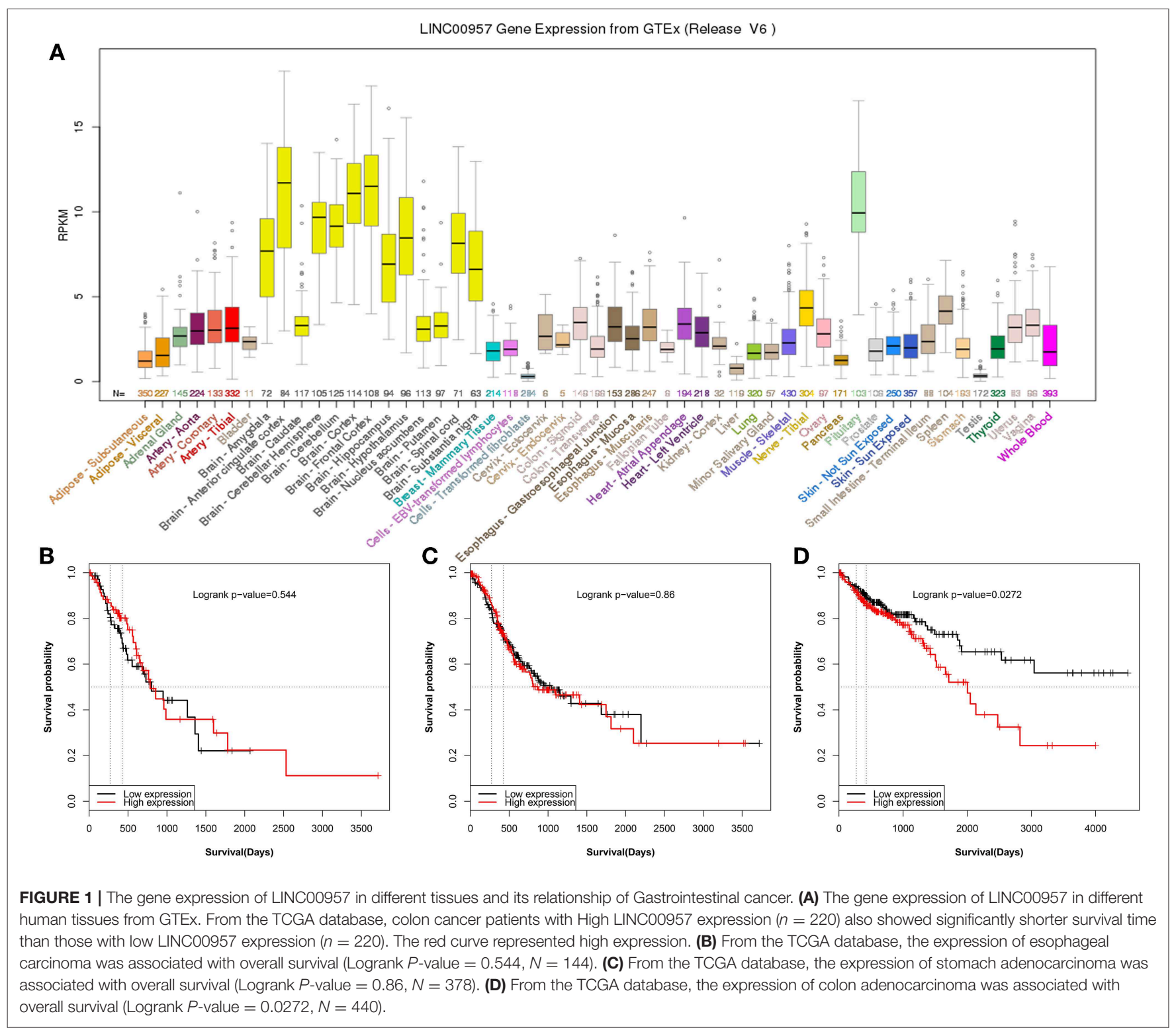

level in Figure $3(P=0.002)$. At the same time, we analyzed the relationship of LINC00957 expression levels and prognosis in CRC patients from TCGA database. LINC00957 expression showed shorter survival time than those with low LINC00957 expression in Figure 1D $(P=0.03)$. We then analyzed the factors of poor prognosis by univariate and multivariate cox proportional hazards analyses. In univariate overall survival (OS) analysis, we observed that LINC00957 expression $(P<0.001)$, TNM stage $(P<0.001)$, chemotherapy outcome $(P=0.014)$ and gender $(P=0.048)$ retained significance as prognostic factors (Table 3). In multivariate OS analysis, we showed that LINC00957 expression $(P=0.010)$ and chemotherapy outcome $(P=0.018)$ were an independent predictor of poor prognosis. Accordingly, our data demonstrated that high expression of LINC00957 could predicate a poor prognosis of patients with CRC.

\section{LINC00957 Expression Was Associated With Drug Resistance by Regulating P-gP}

The two 5-Fluorouracil-resistant model cell lines (LOVO/5-FU and HCT8/5-FU) were used to study the resistance mechanism of LINC00957. Firstly, three pairs of silence primers of LINC00957 were designed to reduce the expression of LINC00957 in both resistant cells. As showed in Figure 4A, the silence effect of siRNA2 in these two cells was the best than siRNA1 and siRNA3, and the result of siRNA2 was remarkably lower expression of LINC00957 comparted with non-silence $(P<$ 0.001). According to the CTB experiment, the $\mathrm{IC}_{50}$ of $\mathrm{LOVO}$ was $428.5 \mathrm{ng} / \mathrm{mL}(\operatorname{LogIC} 50=2.632 \pm 0.051)$; the $\mathrm{IC}_{50}$ of $\mathrm{LOVO} / 5-\mathrm{FU}$ was $1621 \mathrm{ng} / \mathrm{mL}(\operatorname{LogIC} 50=3.210 \pm 0.048)$; the $\mathrm{IC}_{50}$ of $\mathrm{LOVO} / 5-\mathrm{FU} / \mathrm{si}-\mathrm{RNA} 2$ was $590.1 \mathrm{ng} / \mathrm{mL}(\mathrm{LogIC} 50=$ $2.771 \pm 0.061)$. The $\mathrm{IC}_{50}$ of HCT was $409.8 \mathrm{ng} / \mathrm{mL}(\operatorname{LogIC} 50$ $=2.613 \pm 0.052)$; the $\mathrm{IC}_{50}$ of $\mathrm{HCT} / 5-\mathrm{FU}$ was $3064 \mathrm{ng} / \mathrm{mL}$ 

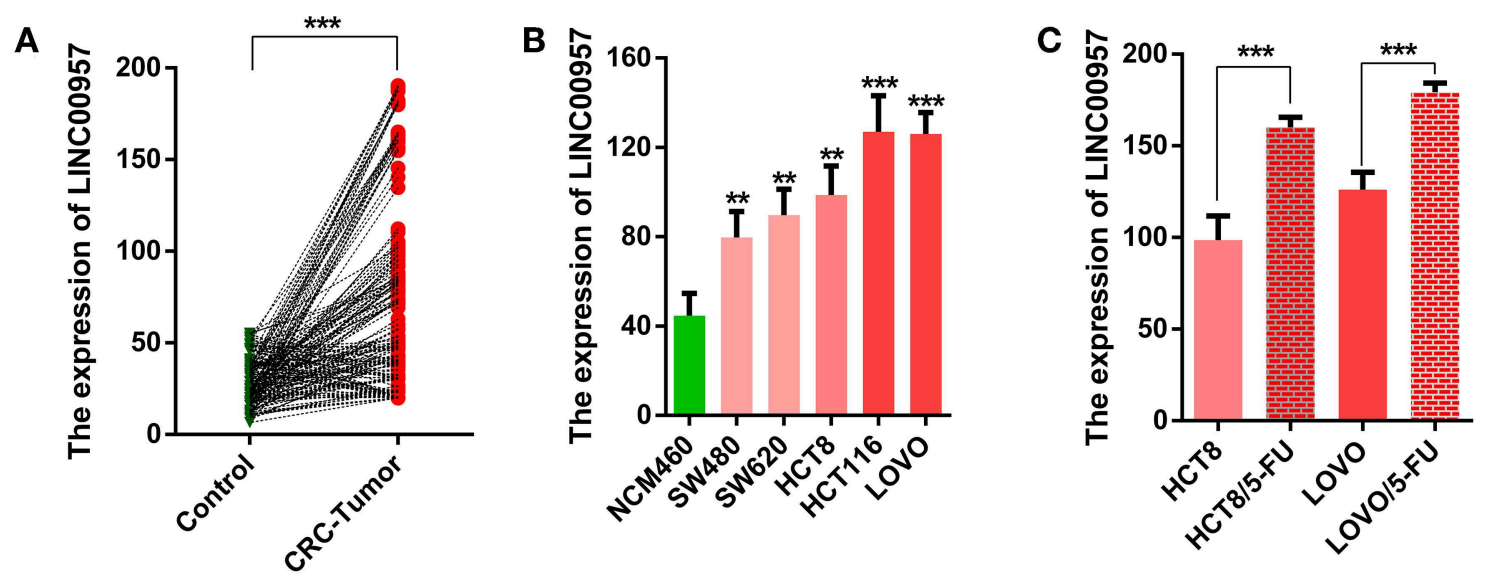

FIGURE 2 | The relative expression level of LINC00957 in tissue samples and cell lines. (A) The green points represented normal tissues as control, while the red stood for CRC. A line connects two pionts (the expression levels of LINC00957 shown in (A) from non-tumor to CRC with an increased trend, suggesting that the expression level of LINC00957 was higher in CRC tissues than in the matched non-tumor tissues. (B) Expression levels of LINC00957 in CRC cell lines including (SW480,SW620,HCT8,LOVO, and HCT116) and the normal human colon epithelial cell line NCM460 was measured by qRT-PCR. (C) The relative expression levels of LINC00957 in CRC cell lines and their matched 5 -FU resistant cell lines were detected by qRT-PCR. ${ }^{* *} P<0.01,{ }^{* * *} P<0.001$.

TABLE 2 | The correlation between LINC00957 expression and clinicopathological variables in CRC patients.

\begin{tabular}{|c|c|c|c|c|c|}
\hline Variable & Number & $\begin{array}{c}\text { Low } \\
\text { expression }\end{array}$ & $\begin{array}{c}\text { High } \\
\text { expression }\end{array}$ & $x^{2}$ test & $P$-value \\
\hline \multicolumn{6}{|l|}{ AGE (YEARS) } \\
\hline$<60$ & 82 & 47 & 35 & 0.078 & 0.874 \\
\hline$\geq 60$ & 78 & 43 & 35 & & \\
\hline \multicolumn{6}{|l|}{ GENDER } \\
\hline Male & 73 & 42 & 31 & 0.09 & 0.873 \\
\hline Female & 87 & 48 & 39 & & \\
\hline \multicolumn{6}{|l|}{ TUMOR SIZE } \\
\hline$<4 \mathrm{~cm}$ & 71 & 37 & 34 & 0.888 & 0.423 \\
\hline$\geq 4 \mathrm{~cm}$ & 89 & 53 & 36 & & \\
\hline \multicolumn{6}{|c|}{ DIFFERENTIATION } \\
\hline Well/moderately & 84 & 46 & 38 & 0.159 & 0.751 \\
\hline Poorly & 76 & 44 & 32 & & \\
\hline \multicolumn{6}{|c|}{ PRIMARY TUMOR LOCATION } \\
\hline Colon cancer & 78 & 41 & 37 & 0.84 & 0.426 \\
\hline Rectal cancer & 82 & 49 & 33 & & \\
\hline \multicolumn{6}{|l|}{ TNM STAGE } \\
\hline |-I|| stage & 116 & 71 & 45 & 4.212 & 0.040 \\
\hline IV stage & 44 & 19 & 25 & & \\
\hline \multicolumn{6}{|c|}{ CHEMOTHERAPY OUTCOME } \\
\hline CR/PR & 117 & 72 & 45 & 4.948 & 0.026 \\
\hline SD/PD & 43 & 18 & 25 & & \\
\hline
\end{tabular}

Bold values indicate $P<0.05$.

$(\operatorname{LogIC} 50=3.486 \pm 0.045)$; the $\mathrm{IC}_{50}$ of $\mathrm{HCT} / 5-\mathrm{FU} / \mathrm{si}-\mathrm{RNA} 2$ was $941.3 \mathrm{ng} / \mathrm{mL}(\operatorname{LogIC50}=2.974 \pm 0.096)$. As shown in Figures 4B,C, inhibition of LINC00957 significantly reversed the 5 -FU resistance in both model cell lines. By low-throughput screening of the drug-resistant relative genes, the transcription level of $\mathrm{ABCB} 1$ gene was significantly down-regulated after
TABLE 3 | Univariate and multivariate analysis of prognostic factors of CRC

\begin{tabular}{|c|c|c|c|c|}
\hline \multirow[t]{2}{*}{ Variable } & \multirow{2}{*}{$\begin{array}{c}\begin{array}{c}\text { Univariate } \\
\text { analysis }\end{array} \\
\text { HR }(95 \% \mathrm{Cl})\end{array}$} & \multicolumn{3}{|c|}{ Multivariate analysis } \\
\hline & & $P$-value & HR (95\%Cl) & $P$-value \\
\hline Age (<60 vs. $60>)$ & $\begin{array}{c}0.997 \\
(0.981-1.012)\end{array}$ & 0.674 & & \\
\hline Gender (Male vs. Female) & $\begin{array}{c}2.002 \\
(1.009-3.989)\end{array}$ & 0.048 & & \\
\hline $\begin{array}{l}\text { Tumer size }(<4 \mathrm{~cm} \text { vs. } \\
>4 \mathrm{~cm})\end{array}$ & $\begin{array}{c}0.965 \\
(0.909-1.026)\end{array}$ & 0.256 & & \\
\hline $\begin{array}{l}\text { Histological grade } \\
\text { (Well/moderately vs. Poorly) }\end{array}$ & $\begin{array}{c}0.874 \\
(0.639-1.195)\end{array}$ & 0.400 & & \\
\hline $\begin{array}{l}\text { Primary tumor location } \\
\text { (colon vs. rectal) }\end{array}$ & $\begin{array}{c}0.846 \\
(0.617-1.159)\end{array}$ & 0.297 & & \\
\hline $\begin{array}{l}\text { TNM stage (IV stage vs. I-III } \\
\text { stage) }\end{array}$ & $\begin{array}{c}1.913 \\
(1.339-2.734)\end{array}$ & $<0.001$ & & \\
\hline $\begin{array}{l}\text { Chemotherapy outcome } \\
\text { (SD/PD vs. CR/PR) }\end{array}$ & $\begin{array}{c}1.844 \\
(1.283-2.650)\end{array}$ & 0.014 & $\begin{array}{c}1.541 \\
(1.066-2.228)\end{array}$ & 0.018 \\
\hline $\begin{array}{l}\text { LINC00957 (High vs. Low } \\
\text { expression) }\end{array}$ & $\begin{array}{c}3.008 \\
(2.051-4.412)\end{array}$ & $<0.002$ & $\begin{array}{c}2.718 \\
(1.839-4.017)\end{array}$ & 0.010 \\
\hline
\end{tabular}

interfering with RNA (Figure 4D). At the same time, the expression of $\mathrm{P}-\mathrm{gP}$ in both cell lines were also significantly down-regulated at the protein level ABCB1 (Figures 4E,F). In clinical samples, LINC00957 was positively associated with P-gP both in in transcription and protein levels (all $P<0.05$, Table 4). These results indicated that LINC00957 was involved in P-gP mediated chemo-resistance.

\section{DISCUSSION}

Colorectal cancer ranks second in terms of mortality and third in terms of incidence, accounting for about $10 \%$ new colorectal 


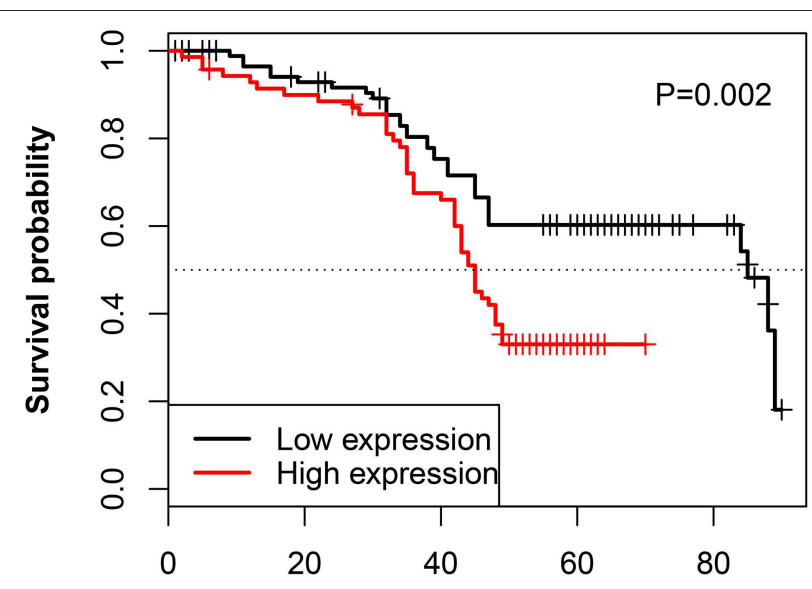

FIGURE 3 | Correlation between LINC00957 expression and OS time in CRC patients. Kaplan-Meier postoperative survival curve for patterns of patients with CRC and LINC00957 expression. In our data, CRC patients with High LINC00957 expression $(n=80)$ showed shorter survival time than those with low LINC00957 expression $(n=80)$.

cancer cases and deaths in $2018(5,17)$. Therefore, it is necessary to identify new molecular targets for the diagnosis, prognosis, and treatment of CRC (18). LincRNAs play vital roles in many biological processes and numerous studies have figured out that aberrant expression of lincRNAs is closely associated with the occurrence and development of malignant tumors (19-21). For instance, lincRNA DQ786243 contributed to proliferation and metastasis of colorectal cancer both in vitro and in vivo (22); GHET1 and TUG1 promoted colon cancer progression $(23,24)$; GAS5 contributed to lymphatic metastasis in colorectal cancer (22); Linc00152 and LincRNA-ROR hold prognostic value $(15,25)$. LncRNAs were reported to be involved in CRC stem cells, and several individual lncRNAs were connected to Wnt signaling through other mechanisms (26). All those reports showed the missing non-coding lincRNAs played vital roles in CRC. However, the role of linc00957 in CRC is still unknown up to now. In order to find interested lincRNA, we analyzed some lincRNAs expression levels using OncoLnc (25), linking TCGA survival data to mRNAs, miRNAs, and lncRNAs (27). We found that patients with higher LINC00957 expression level had dramatically shorter OS in CRC, while in ESAD and STAD are not. it might be that LINC00957 was prognostic marker special for CRC. Therefore, it was necessary to explore its function in CRC.

Firstly, we explored whether LINC00957 was abnormally expressed in CRC. RT-PCR analysis was used to detect the expression of LINC00957 in 160 cases of the cancer and adjacent tissues. The results showed that LINC00957 was highly expressed in the cancer, and the same result was also found in cell lines. These data indicated that LINC00957 was associated with CRC. Secondly, we explored the correlation between the expression of LINC00957 and its clinicopathological variables. These results revealed that its high expression was significantly associated with TNM advanced stage, poor chemotherapy outcomes and worse prognosis. It revealed that the expression of LINC00957 was involved in tumor progression and multidrug resistance, and could be an independent prognostic indicator. Some references had reported that LINC-VLDLR and LINC-ROR were associated drug resistance $(28,29)$. Therefore, we speculated that LINC00957 might affect poor prognosis through inducing chemo-resistance. To test the hypothesis, we performed two 5-FU resistant cell lines (LOVO/5-FU and HCT8/5-FU) as chemo-resistance model cells. It was found that LINC00957 was remarkably higher expression in these chemo-resistance model cells than relative sensitive cell lines (LOVO and HCT8).Inhibition of its expression enhanced sensitivity and reversed 5-FU resistance. In the mechanism, we speculated that the relative multi-drug genes were associated with LINC00957 expression. P-gp(known as multidrug resistance protein 1 and encoded by the ABCB1 gene) efflux activity is capable of lowering intracellular concentrations of otherwise beneficial compounds, such as chemotherapeutics and other medications, to subtherapeutic levels (30). Consequently, P-gp overexpression is one of the main mechanisms behind decreased intracellular drug accumulation and development of multidrug resistance in human multidrug-resistant (MDR) cancers (31). Through low throughput screening, only $\mathrm{P}-\mathrm{gP}$ expression was down-regulated after inhibiting the expression of LINC00957.It indicated that LINC00957 might reverse 5-FU resistance by regulating $\mathrm{P}-\mathrm{gP}$ expression. To further explore this mechanism, we enriched KEGG pathway by GSEA from TCGA database. The Notch signaling pathway was significantly enriched in LINC00957 hi group. It suggested that LINC00957 might induce multidrug resistance by activate Notch signaling pathway. Recently, emerging evidences suggest that Notch signaling pathway is one of the most important signaling pathways in drug-resistant tumor cells (32). The activated Notch signaling contributes to resistance in enzalutamide-resistant prostate cancer cells (33). Inhibition of Notch signaling reverses docetaxel resistance in prostate cancer (34).

These findings demonstrated that the long non-coding RNA 00957 (LINC00957) expression as a novel prognostic biomarker in colorectal cancer (CRC). Aberrant expression of LINC00957 was associated with CRC in GTEx dataset, and his upregulation was confirmed by qRT-PCR in patients' tumor and paired normal tissues. Expression of LINC00957 was significantly associated with advanced TNM stage, poor chemotherapy outcomes, and worse prognosis. Thus, we speculated that LINC00957 was involved in tumor progression and development of multidrug resistance. The latter hypothesis was partially demonstrated using two 5-Fluorouracil-resistant model cell lines (LOVO/5-FU and HCT8/5-FU), in which the silencing of LINC00957 was able to reverse 5-FU resistance, potentially through $\mathrm{P}-\mathrm{gP}$ modulation. Beyond the functional study using knockdown experiments, this study also shows the potential role of the linc00975 in drug resistance. All these data together suggest a role of LINC00957 as oncogenic gene in CRC. Overall, this study is novel and wellperformed, representing a good starting point for the exploration of the role of LINC00975 in CRC. The results obtained from CRC patients' analysis are a reasonable base for further experiments.

This research had some limitations. Firstly, the study is very linear, clear and simple. However, the evidences supporting the 


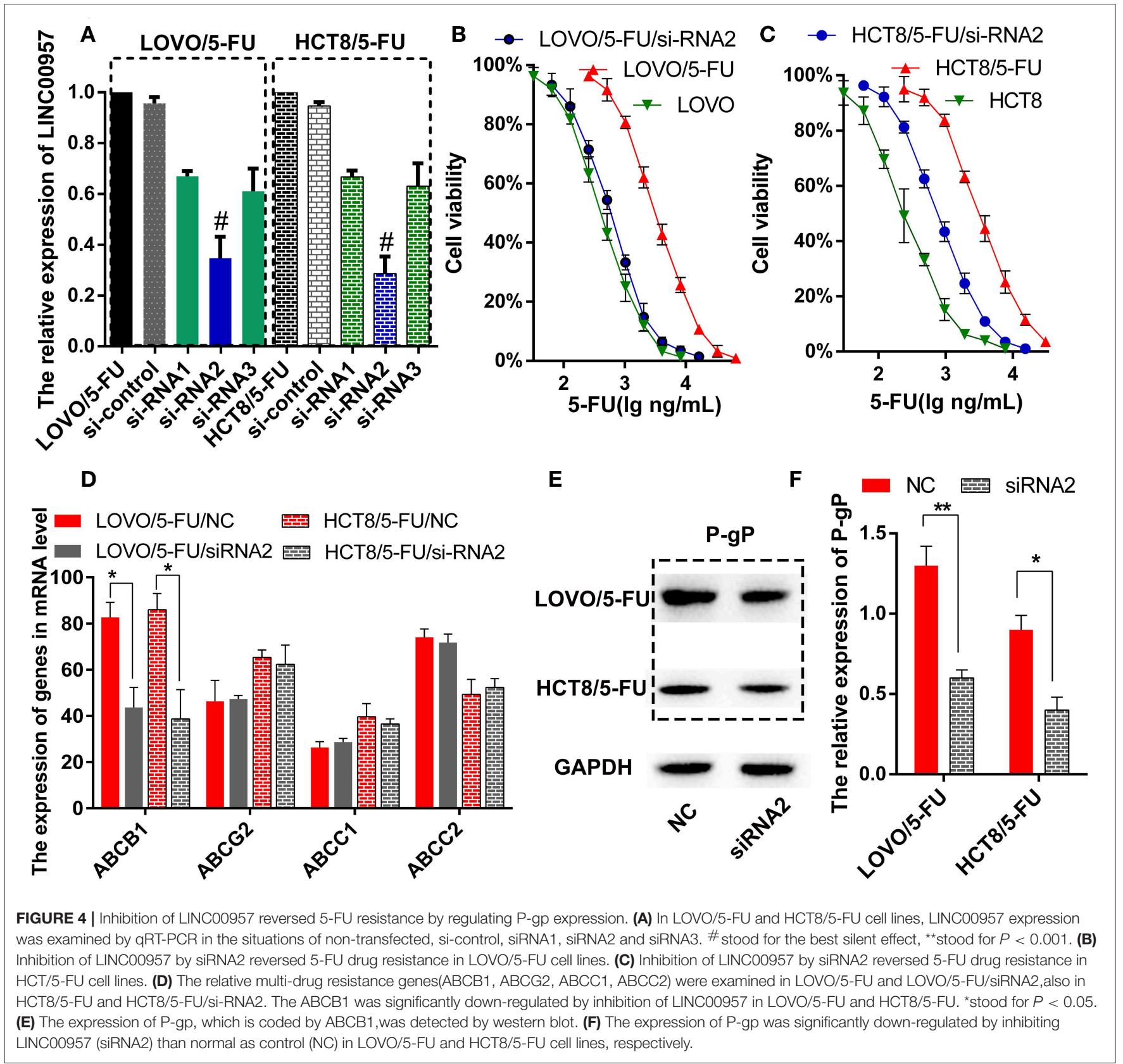

TABLE 4 | The correlation of LINC00957 with P-gp both in transcription and protein levels in clinical samples.

\begin{tabular}{|c|c|c|c|c|c|}
\hline Variable & Number & LINC00957 high & LINC00957 low & $x^{2}$ test & $P$-value \\
\hline ABCB1 high & 62 & 34 & 28 & 4.349 & 0.037 \\
\hline ABCB1 low & 98 & 36 & 62 & & \\
\hline P-gP high & 69 & 38 & 31 & 5.536 & 0.019 \\
\hline P-gP low & 91 & 32 & 59 & & \\
\hline
\end{tabular}

involvement of LINC00975 overexpression in the development of a drug-resistance phenotype are poor. Additional and more pround experiments are needed to sustain with mechanistic evidences, the designation of LINC00975 expression as a potential therapeutic target for CRC. Secondly, all experiments have been performed well and reasonably. Although the functional mechanism of linc00975 in CRC development and drug-resistance have not been investigated, it could be continued in the further studies.

\section{DATA AVAILABILITY}

The raw data supporting the conclusions of this manuscript will be made available by the authors, without undue reservation, to any qualified researcher. 


\section{AUTHOR CONTRIBUTIONS}

DH and LZ designed the study. LZ and LL developed the methodology, performed the analysis, and wrote the manuscript. PZ, YC, and LL collected the data, cultured cell lines and did PCR, CTB, Western blot assays.

\section{REFERENCES}

1. Sinicrope FA. Lynch syndrome-associated colorectal cancer. $N$ Engl J Med. (2018) 379:764-73. doi: 10.1056/NEJMcp1714533

2. Chen W, Zheng R, Baade PD, Zhang S, Zeng H, Bray F, et al. Cancer statistics in China, 2015. CA Cancer J Clin. (2016) 66:115-32. doi: 10.3322/caac. 21338

3. Dienstmann R, Mason MJ, Sinicrope FA, Phipps AI, Tejpar S, Nesbakken A, et al. Prediction of overall survival in stage II and III colon cancer beyond TNM system: a retrospective, pooled biomarker study. Ann Oncol. (2017) 28:1023-31. doi: 10.1093/annonc/mdx052

4. Suenaga M, Akiyoshi T, Shinozaki E, Fujimoto Y, Matsusaka S, Konishi T, et al. A feasibility study of capecitabine and oxaliplatin for patients with stage II/III colon cancer -ACTOR Study. Anticancer Res. (2018) 38:17417. doi: 10.21873 /anticanres. 12410

5. Tutino V, Defrancesco ML, Tolomeo M, DE Nunzio V, Lorusso D, Paleni $\mathrm{D}$, et al. The expression of riboflavin transporters in human colorectal cancer. Anticancer Res. (2018) 38:2659-67. doi: 10.21873/anticanres. 12508

6. Lea MA, Kim H, Des BC. Effects of biguanides on growth and glycolysis of bladder and colon cancer cells. Anticancer Res. (2018) 38:500311. doi: 10.21873/anticanres.12819

7. Tei M, Otsuka M, Suzuki Y, Kishi K, Tanemura M, Akamatsu H. Safety and feasibility of single-port surgery for colon cancer in octogenarians. Anticancer Res. (2018) 38:2967-2972. doi: 10.21873/anticanres.12547

8. Dai W, Feng Y, Mo S, Xiang W, Li Q, Wang R, et al. Transcriptome profiling reveals an integrated mRNA-lncRNA signature with predictive value of early relapse in colon cancer. Carcinogenesis. (2018) 39:123544. doi: 10.1093/carcin/bgy087

9. Wang Y, Ding X, Hu H, He Y, Lu Z, Wu P, et al. Long non-coding RNA lnc-PCTST predicts prognosis through inhibiting progression of pancreatic cancer by downregulation of TACC-3. Int J Cancer. (2018) 143:314354. doi: 10.1002/ijc.31657

10. Ransohoff JD, Wei Y, Khavari PA. The functions and unique features of long intergenic non-coding RNA. Nat Rev Mol Cell Biol. (2018) 19:14357. doi: $10.1038 / \mathrm{nrm} .2017 .104$

11. Wang ZK, Yang L, Wu LL, Mao H, Zhou YH, Zhang PF, et al. Long noncoding RNA LINC00261 sensitizes human colon cancer cells to cisplatin therapy. Braz J Med Biol Res. (2017) 51:e6793. doi: 10.1590/1414-431x20 176793

12. Yang L, Tang Y, He Y, Wang Y, Lian Y, Xiong F, et al. High expression of LINC01420 indicates an unfavorable prognosis and modulates cell migration and invasion in nasopharyngeal carcinoma. J Cancer. (2017) 8:97103. doi: 10.7150/jca.16819

13. Gao X, Wen J, Gao P, Zhang G, Zhang G. Overexpression of the long noncoding RNA, linc-UBC1, is associated with poor prognosis and facilitates cell proliferation, migration, and invasion in colorectal cancer. Onco Targets Ther. (2017) 10:1017-26. doi: 10.2147/OTT.S129343

14. Yu T, Shan TD, Li JY, Huang CZ, Wang SY, Ouyang H, et al. Knockdown of linc-UFC1 suppresses proliferation and induces apoptosis of colorectal cancer. Cell Death Dis. (2016) 7:e2228. doi: 10.1038/cddis. 2016.124

15. Zhou P, Sun L, Liu D, Liu C, Sun L. Long non-coding RNA lincRNAROR promotes the progression of colon cancer and holds prognostic value by associating with miR-145. Pathol Oncol Res. (2016) 22:73340. doi: 10.1007/s12253-016-0061-x

\section{FUNDING}

The Natural Science Foundation of Jiangsu Province (No. BK20171150), the National Natural Science Foundation of China (No. 81502042), Research Project of Health and Family Planning Commission of Wuxi (No. Q201758) and Nanchang Hongda Jianghua Educational Foundation.

16. Marín-Béjar O, Mas AM, González J, Martinez D, Athie A, Morales $\mathrm{X}$, et al. The human IncRNA LINC-PINT inhibits tumor cell invasion through a highly conserved sequence element. Genome Biol. (2017) 18:202. doi: 10.1186/s13059-017-1331-y

17. Virgilio E, Giarnieri E, Giovagnoli MR, Montagnini M, Proietti A, D’Urso $\mathrm{R}$, et al. Gastric cancer cells in peritoneal lavage fluid: a systematic review comparing cytological with molecular detection for diagnosis of peritoneal metastases and prediction of peritoneal recurrences. Anticancer Res. (2018) 38:1255-62. doi: 10.21873/anticanres.12347

18. Nakamura M, Suetsugu A, Hasegawa K, Satake T, Kunisada T, Shimizu $\mathrm{M}$, et al. Color-coded imaging distinguishes cancer cells, stromal cells, and recombinant cancer-stromal cells in the tumor microenvironment during metastasis. Anticancer Res. (2018) 38:4417-23. doi: 10.21873/anticanres.12743

19. Gong J, Zhang H, He L, Wang L, Wang J. Increased expression of long non-coding RNA BCAR4 is predictive of poor prognosis in patients with non-small cell lung cancer. Tohoku J Exp Med. (2017) 241:2934. doi: 10.1620/tjem.241.29

20. Quan Y, Song K, Zhang Y, Zhu C, Shen Z, Wu S, et al. Roseburia intestinalisderived flagellin is a negative regulator of intestinal inflammation. Biochem Biophys Res Commun. (2018) 501:791-99. doi: 10.1016/j.bbrc.2018.05.075

21. Huang $\mathrm{Y}$, Hu Y, Jin Z, Shen Z. LncRNA snaR upregulates GRB2-associated binding protein 2 and promotes proliferation of ovarian carcinoma cells. Biochem Biophys Res Commun. (2018) 503:2028-32. doi: 10.1016/j.bbrc.2018.07.152

22. Sun L, Xue H, Jiang C, Zhou H, Gu L, Liu Y, et al. LncRNA DQ786243 contributes to proliferation and metastasis of colorectal cancer both in vitro and in vivo. Biosci Rep. (2016) 36:e00328. doi: 10.1042/BSR20160048

23. Zhou J, Li X, Wu M, Lin C, Guo Y, Tian B. Knockdown of long noncoding RNA GHET1 inhibits cell proliferation and invasion of colorectal cancer. Oncol Res. (2016) 23:303-9. doi: 10.3727/096504016X14567549091305

24. Zhai HY, Sui MH, Yu X, Qu Z, Hu JC, Sun HQ, et al. Overexpression of long non-coding RNA TUG1 promotes colon cancer progression. Med Sci Monit. (2016) 22:3281-7. doi: 10.12659/MSM.897072

25. Yue B, Cai D, Liu C, Fang C, Yan D. Linc00152 functions as a competing endogenous RNA to confer oxaliplatin resistance and holds prognostic values in colon cancer. Mol Ther. (2016) 24:2064-77. doi: 10.1038/mt.2016.180

26. Shen P, Pichler M, Chen M, Calin GA, Ling H. To wnt or lose: the missing non-coding linc in colorectal cancer. Int J Mol Sci. (2017) 18:E2003. doi: 10.3390/ijms18092003

27. Anaya J. OncoLnc: linking TCGA survival data to mRNAs, miRNAs, and lncRNAs. PeerJ Comp Sci. (2016) 2:e67. doi: 10.7717/peerj-cs.67

28. Chen Y, Liu L, Li J, Du Y, Wang J, Liu J. Effects of long noncoding RNA (linc-VLDLR) existing in extracellular vesicles on the occurrence and multidrug resistance of esophageal cancer cells. Pathol Res Pract. (2019) 215:470-77. doi: 10.1016/j.prp.2018.12.033

29. Li C, Zhao Z, Zhou Z, Liu R. Linc-ROR confers gemcitabine resistance to pancreatic cancer cells via inducing autophagy and modulating the miR-124/PTBP1/PKM2 axis. Cancer Chemother Pharmacol. (2016) 78:1199207. doi: 10.1007/s00280-016-3178-4

30. Sui H, Fan ZZ, Li Q. Signal transduction pathways and transcriptional mechanisms of ABCB1/Pgp-mediated multiple drug resistance in human cancer cells. J Int Med Res. (2012) 40:426-35. doi: 10.1177/147323001204000204

31. Breier A, Gibalova L, Seres M, Barancik M, Sulova Z. New insight into pglycoprotein as a drug target. Anti-Cancer Agents Med Chem. (2013) 13:15970. doi: $10.2174 / 187152013804487380$ 
32. Wang Z, Li Y, Ahmad A, Azmi AS, Banerjee S, Kong D, et al. Targeting notch signaling pathway to overcome drug resistance for cancer therapy. Biochim Biophys Acta. (2010) 1806:258-67. doi: 10.1016/j.bbcan.2010. 06.001

33. Farah E, Li C, Cheng L, Kong Y, Lanman NA, Pascuzzi P, et al. $\mathrm{NOTCH}$ signaling is activated in and contributes to resistance in enzalutamide-resistant prostate cancer cells. J Biol Chem. (2019) 294:854354. doi: 10.1074/jbc.RA118.006983

34. Qiu S, Deng L, Bao Y, Jin K, Tu X, Li J, et al. Reversal of docetaxel resistance in prostate cancer by Notch signaling inhibition. Anticancer Drugs. (2018) 29:871-79. doi: 10.1097/CAD.0000000000000659
Conflict of Interest Statement: The authors declare that the research was conducted in the absence of any commercial or financial relationships that could be construed as a potential conflict of interest.

Copyright (C) 2019 Zhang, Li, Zhang, Cai and Hua. This is an open-access article distributed under the terms of the Creative Commons Attribution License (CC BY). The use, distribution or reproduction in other forums is permitted, provided the original author(s) and the copyright owner(s) are credited and that the original publication in this journal is cited, in accordance with accepted academic practice. No use, distribution or reproduction is permitted which does not comply with these terms. 